\title{
Non-Linear Optimization for Multi-Path Source Routing in OBS Networks
}

\author{
Mirosław Klinkowski, Student Member, IEEE, Michał Pióro, Senior Member, IEEE, \\ Davide Careglio, Member, IEEE, Marian Marciniak, Senior Member, IEEE, and Josep Solé-Pareta
}

\begin{abstract}
Optical Burst Switching (OBS) architectures without buffering capabilities are sensitive to burst losses. A proper routing strategy may help to reduce such congestion. In this letter we present a novel approach for OBS multi-path source routing problem. Our solution is based on non-linear optimization with a straightforward calculation of partial derivatives. Simulation results demonstrate that optimized routing effectively reduces the overall burst loss probability with respect to shortest path and deflection routing.
\end{abstract}

Index Terms - Network optimization, optical burst switching, routing.

\section{INTRODUCTION}

$\mathbf{O}$ PTICAL Burst Switching is a photonic network technology aiming at efficient transport of IP traffic [1]. OBS architectures without buffering capabilities are sensitive to burst congestion. A burst loss probability (BLP) which adequately represents the congestion state of entire network is the primary metric of interest in an OBS network. The network congestion can be reduced by using proper routing; in this context deflection routing (e.g., see [2]), a common routing strategy in OBS, has been considered. Although deflection routing improves network performance under low traffic loads, still, it may increase burst losses under moderate and high loads.

In this letter we consider another approach - multi-path source routing - and we use network optimization theory to improve it. Since an overall BLP has a non-linear character (see e.g., [3]), either linear programming formulations with piecewise linear approximations of this function (see e.g., [4]) or non-linear optimization gradient methods [5] can be used.

In our non-linear optimization problem we assume that there is a pre-established virtual path topology consisting of a limited number of paths between each pair of source-destination nodes. Using a gradient optimization method we calculate a traffic splitting vector that determines the distribution of traffic over these paths. The proposed solution can be used, in particular, for static (pre-planed) routing, where the traffic distribution is calculated based on a given (long-term) traffic demand matrix. Then either a periodic or a threshold-triggered

Manuscript received August 7, 2007. The associate editor coordinating the review of this letter and approving it for publication was Prof. Nasir Ghani.

M. Klinkowski, D. Careglio and J. Solé-Pareta are with the Technical University of Catalonia, Spain (e-mail: \{mklinkow, careglio, pareta\}@ac.upc.edu).

M. Pióro is with the Warsaw University of Technology, Poland and the Lund University, Sweden (e-mail: mpp@tele.pw.edu.pl).

M. Klinkowski and M. Marciniak are with the National Institute of Telecommunications, Poland (e-mail: mmarcin@itl.waw.pl).

Digital Object Identifier 10.1109/LCOMM.2007.071311.

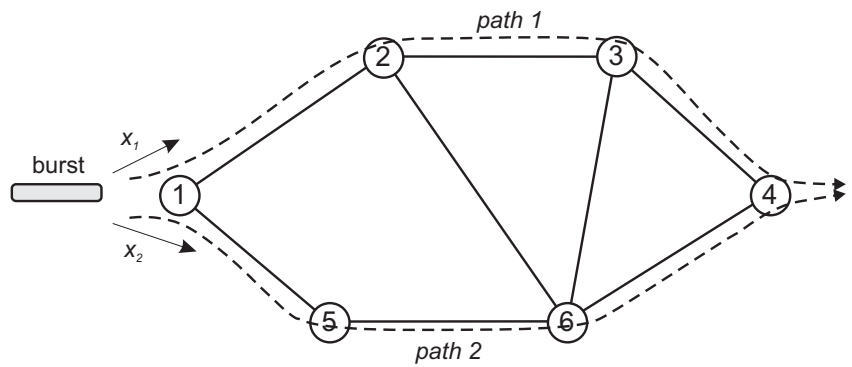

Fig. 1. Example of OBS network with source-based routing; $x_{1}$ and $x_{2}$ are the traffic splitting factors and $x_{1}+x_{2}=1$.

update of the splitting vector can be performed if the traffic demand matrix is subject to a change.

\section{ROUTING SCENARIO}

We use $\mathcal{G}=(\mathcal{V}, \mathcal{E})$ to denote the graph of an OBS network; the set of nodes is denoted as $\mathcal{V}$, and the set of links is denoted as $\mathcal{E}$. Link $e \in \mathcal{E}$ comprises $C_{e}$ wavelengths. $\mathcal{P}$ defines a set of all paths predefined between each source nodes $s$ and destination nodes $d$, where $s, d \in \mathcal{V}$ and $s \neq d$. Each individual path $p \in \mathcal{P}$ is identified with a subset $p \subseteq \mathcal{E}$. Subset $P_{s d} \subseteq \mathcal{P}$ identifies all paths from source $s$ to destination $d$. Subset $Q_{e} \subseteq \mathcal{P}$ identifies all paths that go through link $e$.

We assume that the network applies source-based routing, so that the source node determines the path of a burst that enters the network (see Fig. 1). Moreover, the network uses multi-path routing where each subset $P_{s d}$ comprises a (small) number of paths and a burst can follow one of them. Path selection is performed according to given traffic splitting factor $x_{p}$, such that

$$
\begin{gathered}
0 \leq x_{p} \leq 1, \quad p \in \mathcal{P}, \\
\sum_{p \in P_{s d}} x_{p}=1, \quad s, d \in \mathcal{V}, s \neq d .
\end{gathered}
$$

The reservation (holding) times on each link are i.i.d. random variables with the mean equal to the mean burst duration $h$; for simplicity we assume $h=1$. The demand traffic pattern is described by matrix $\left[t_{s d}\right]_{s, d \in \mathcal{V}}$ and bursts destined to given node $d$ arrive at node $s$ according to a Poisson process of (long-term) rate $t_{s d} / h=t_{s d}$. Thus traffic $v_{p}$ offered to path $p \in P_{s d}$ can be calculated as

$$
v_{p}=x_{p} \tau_{p},
$$

where $\tau_{p}=t_{s d}$ is the total traffic offered between $s$ and $d$.

Here vector $\boldsymbol{x}=\left(x_{1}, \ldots, x_{|\mathcal{P}|}\right)$ determines the distribution of traffic over the network; this vector should be optimized to reduce congestion and to improve overall performance. 


\section{FORMULATION}

\section{A. A loss model of OBS network}

A loss model of OBS network based on the Erlang fixedpoint approximation was proposed by Rosberg et al. [3]. In particular, the traffic offered to link $e$ is obtained as a sum of the traffic offered to all the paths that cross this link reduced by the traffic lost in the preceding links along these paths

$$
\rho_{e}=\sum_{p \in Q_{e}} v_{p} \prod_{g \in r_{p e}}\left(1-E_{g}\right), \quad e \in \mathcal{E}
$$

where subset $r_{p e} \subset p$ identifies all links that precede link $e$ along path $p$.

The formulation of [3] may bring some difficulty in the context of computation of partial derivatives for optimization purposes. Therefore we propose a simplified non-reduced link load model where the traffic offered to link $e$ is calculated as a sum of the traffic offered to all the paths that cross this link

$$
\rho_{e}=\sum_{p \in Q_{e}} v_{p}, \quad e \in \mathcal{E}
$$

The rationale behind this assumption is that under low link losses $E_{g}$, observed in a properly dimensioned network, model (4) can be approximated by (5).

The main modelling steps include the calculation of

1) burst loss probabilities $E_{e}$ on links, given by the Erlang loss formula

$$
E_{e}=E\left(\rho_{e}, C_{e}\right)=\frac{\rho_{e}^{C_{e}}}{C_{e} !}\left[\sum_{i=0}^{C_{e}} \frac{\rho_{e}^{i}}{i !}\right]^{-1}, \quad e \in \mathcal{E}
$$

2) loss probabilities $L_{p}$ of bursts offered to paths

$$
L_{p}=1-\prod_{e \in p}\left(1-E_{e}\right), \quad p \in \mathcal{P}
$$

3) and the overall burst loss probability $B$

$$
B=\sum_{p \in \mathcal{P}} v_{p} L_{p}\left[\sum_{p \in \mathcal{P}} v_{p}\right]^{-1} .
$$

\section{B. Optimization problem}

From equations (3) and (8) we define a cost function to be the subject of optimization:

$$
B(\boldsymbol{x})=\sum_{p \in \mathcal{P}} x_{p} \tau_{p} L_{p} .
$$

The optimization problem is formulated as follows:

$$
\min B(\boldsymbol{x})
$$

subject to the constraints given by (1) and (2).

Since the overall BLP is a non-linear function of vector $\boldsymbol{x}$ the cost function is non-linear as well. Taking into account the form of constraints (5), a particularly convenient optimization method is the Frank-Wolfe reduced gradient method (algorithm 5.10 in [6]); this algorithm was used for a similar problem in circuit-switched (CS) networks [5].

\section{Partial derivatives}

Gradient methods need to employ the calculation of partial derivatives of the cost function. The partial derivative of $B$ with respect to $x_{q}, q \in \mathcal{P}$, could be derived directly from formulae (5)-(8) by a standard method involving resolution of a system of linear equations. Such a computation, however, would be time-consuming. Therefore instead we apply the following exact derivation that is based on the F. Kelly approach proposed for CS networks in [7].

We shall write $f=f(v ; E ; C)$ when we wish to emphasize the functional dependence of a function $f$ on the system parameters $v=\left(v_{p}: p \in \mathcal{P}\right), E=\left(E_{e}: e \in \mathcal{E}\right)$, and $C=\left(C_{e}: e \in \mathcal{E}\right)$.

For each link $e \in \mathcal{E}$ define

$$
\eta_{e}=E\left(\rho_{e}, C_{e}-1\right)-E\left(\rho_{e}, C_{e}\right) .
$$

From (6) it follows that

$$
\frac{d}{d \rho_{e}} E\left(\rho_{e}, C_{e}\right)=\left[1-E\left(\rho_{e}, C_{e}\right)\right] \eta_{e}, \quad e \in \mathcal{E} .
$$

From this and (5), for each path $q$

$$
\frac{\partial}{\partial v_{q}} E_{e}(v ; C)=\left(1-E_{e}\right) \eta_{e}, \quad e \in q .
$$

Define

$$
B(v ; E ; C)=\sum_{p \in \mathcal{P}} v_{p}\left(1-\prod_{g \in p}\left(1-E_{g}\right)\right) .
$$

We find that

$$
\frac{\partial}{\partial v_{q}} B(v ; E ; C)=1-\prod_{g \in q}\left(1-E_{g}\right)=L_{q}, \quad q \in \mathcal{P}
$$

and

$\frac{\partial}{\partial E_{e}} B(v ; E ; C)=\left(1-E_{e}\right)^{-1} \sum_{p \in Q_{e}} v_{p}\left(1-L_{p}\right), \quad e \in \mathcal{E}$.

For each link $e \in \mathcal{E}$ define $c_{e}$ :

$$
c_{e}=\eta_{e} \sum_{p \in Q_{e}} v_{p}\left(1-L_{p}\right) .
$$

From the above, for each path $q \in \mathcal{P}$

$$
\begin{aligned}
\frac{\partial}{\partial v_{q}} B(v ; C) & =\frac{\partial}{\partial v_{q}} B(v ; E(v ; C) ; C) \\
& =\left[\frac{\partial}{\partial v_{q}}+\sum_{e \in q} \frac{\partial}{\partial v_{q}} E_{e}(v ; C) \frac{\partial}{\partial E_{e}}\right] B(v ; E ; C) \\
& =L_{q}+\sum_{e \in q} c_{e} .
\end{aligned}
$$

Finally, because of (3), for each path $q \in \mathcal{P}$ we have

$$
\frac{\partial}{\partial x_{q}} B(\boldsymbol{x})=\tau_{p}\left[L_{q}+\sum_{e \in q} c_{e}\right] \text {. }
$$

Due to assumption (5) we have managed to simplify the model described in [7] and make the calculation of partial derivatives defined by (17) and (19) straightforward, not 


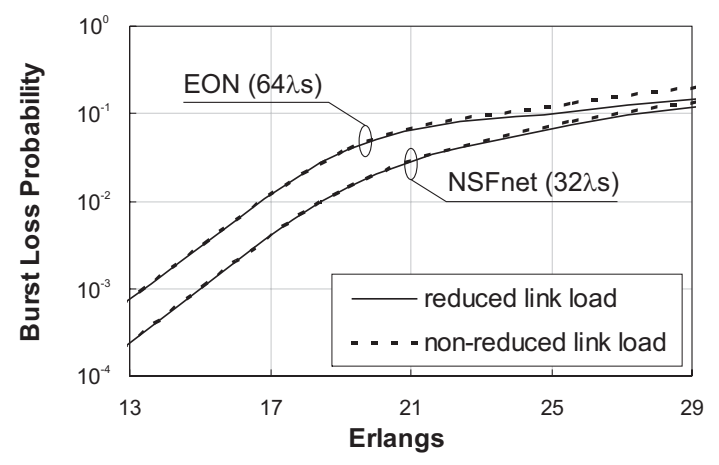

Fig. 2. Accuracy of non-reduced link load model.

involving any iterations. Indeed once $|\mathcal{E}|$ unknowns $\left(c_{e}\right)$ are pre-calculated then they can be used in (19) to obtain the partial derivatives. The calculation of gradient in our method, therefore, is not longer an issue.

Although we are not able to prove that the (not unique) solution is optimal in a globally sense, numerical results show that several repetitions of the optimization of (9) using formula (19) always give us the same (with a finite numerical precision) near-optimal value of $B$.

In order to gain insight into the character of function $B$ we calculate it for vector $\boldsymbol{x}_{0}(\gamma)$, such that:

$$
\boldsymbol{x}_{0}(\gamma)=\gamma \boldsymbol{x}_{1}+(1-\gamma) \boldsymbol{x}_{2}
$$

where $\boldsymbol{x}_{1}$ and $\boldsymbol{x}_{2}$ are two (different) near-optimal vectors, and $\gamma \in[0,1]$.

Numerical results show that $B\left(\boldsymbol{x}_{0}(\gamma)\right)$ is a monotonic function of near-horizontal character.

\section{Results}

We evaluated the performance of our routing scheme in an event-driven simulator. In order to find a splitting vector $\boldsymbol{x}$ specifying a near-optimal routing we use a solver fmincon for constrained nonlinear multivariable functions available in the Matlab environment. Then we apply this vector in the simulator.

The evaluation is performed for NSFnet (15 nodes, 23 links) and EON (28 nodes, 39 links) network topologies; different number of wavelengths $(\lambda s)$ per link are considered, transmission bitrate is 10 Gbps. The optimized routing (OR) is compared with two other routing strategies: a simple shortest path routing (SP) and a pure deflection routing (DR). We consider 2 shortest paths per each source-destination pair of nodes; they are not necessarily disjoint. In SP routing only 1 path is available. Uniform traffic matrix and exponential burst inter-arrivals and durations are considered. All the simulation results have $99 \%$ level of confidence.

In Fig. 2 we compare the overall burst loss probability $B$ of both reduced link loss model (4) and non-reduced link loss model (5), calculated in the function of offered traffic load, which is expressed in Erlangs and normalized to the bitrate (e.g., 10Erlangs mean that each node generates 100Gbps), and with SP routing. We can see that the accuracy of nonreduced link load model is very strict for $B$ below $10^{-2}$.

In Fig. 3 we show $B$ as a function of offered traffic load for different routing scenarios. We see that the optimized routing
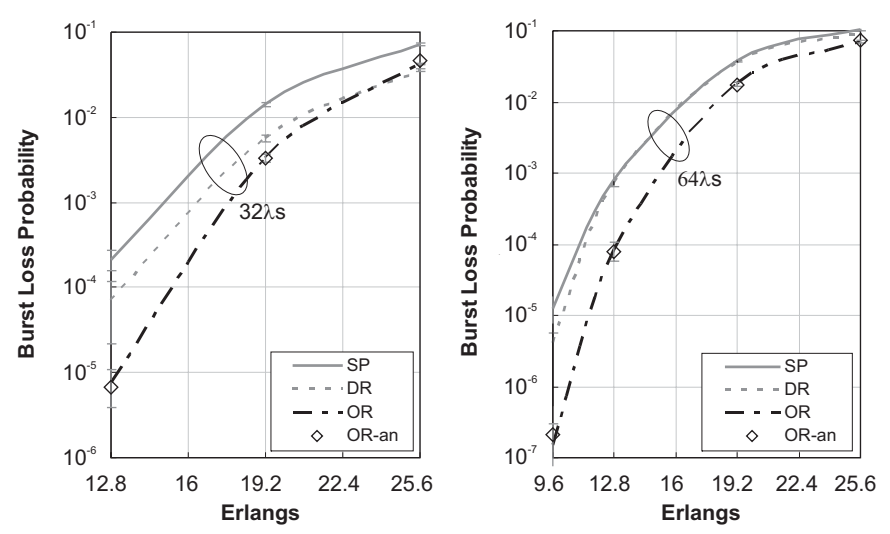

Fig. 3. Comparison of routing schemes a) NSFnet, b) EON.

can achieve very low losses, particularly, when compared with the shortest path routing. Analytical results ('OR-an' in the figure) obtained with (8) correspond very well to simulation results. The optimization takes about $40 \mathrm{sec}$ and $1800 \mathrm{sec}$ for NSFnet network (of 420 paths) and EON network (of 1512 paths), respectively, when using a non-commercial Matlab solver on a Pentium $\mathrm{D}, 3 \mathrm{GHz}$ computer.

\section{Conclusion}

In this letter we have proposed a non-linear optimization method for multi-path source routing problem in OBS networks. In our method we calculate a traffic splitting vector that determines a near-optimal distribution of traffic over routing paths. The presented formulae for partial derivatives are straightforward and very fast to compute. It makes the proposed non-linear optimization method a viable alternative for linear programming formulations based on piecewise linear approximations.

The simulation results demonstrate that our method effectively distributes the traffic over the network and the networkwide burst loss probability can be reduced compared with the shortest path routing.

\section{ACKNOWLEDGEMENT}

This work was supported by the COST 293 and the Spanish Ministry of Education and Science under the CATARO project (Ref. TEC2005-08051-C03-01).

\section{REFERENCES}

[1] C. Qiao and M. Yoo, "Optical burst switching (OBS)-a new paradigm for an optical Internet," J. High Speed Networks, vol. 8, no. 1, pp. 69-84, Mar. 1999.

[2] C. Cameron, et al., "Prioritized deflection routing in optical burst switching networks," IEICE Trans. Commun., vol. E88-B, no. 5, pp. 1861-1867, May 2005.

[3] Z. Rosberg, et al., "Performance analyses of optical burst switching networks," IEEE J. Select. Areas Commun., vol. 21, no. 7, pp. 11871197, Sept. 2003.

[4] J. Teng and G. Rouskas, "Traffic engineering approach to path selection in optical burst switching networks," J. Opt. Net., vol. 4, no. 11, 2005.

[5] R. J. Harris, "The modified reduced gradient method for optimally dimensioning telephone networks," Australian Telecommun. Research, vol. 10, no. 1, pp. 30-35, 1976.

[6] M. Pioro and D. Medhi, Routing, Flow, and Capacity Design in Communication and Computer Networks. Morgan Kaufmann, 2004.

[7] F. P. Kelly, "Routing in circuit-switched networks: optimization, shadow prices and decentralization," Advanced Applied Probability, vol. 20, pp. 112-144, 1988. 